\title{
Uncovering of the Association Between m5C Regulator-Mediated Methylation Modification Patterns and Tumor Microenvironment Infiltration Characteristics in Hepatocellular Carcinoma
}

\section{Xinyu Gu}

The First Affiliated Hospital, College of Medicine, Zhejiang University, Hangzhou, Zhejiang 310003, China

Haibo Zhou

The First Affiliated Hospital, College of Medicine, Zhejiang University, Hangzhou, Zhejiang 310003, China

\section{Qingfei Chu}

The First Affiliated Hospital, College of Medicine, Zhejiang University, Hangzhou, Zhejiang 310003, China

\section{Qiuxian Zheng}

The First Affiliated Hospital, College of Medicine, Zhejiang University, Hangzhou, Zhejiang 310003, China

\section{Jing Wang}

The First Affiliated Hospital, College of Medicine, Zhejiang University, Hangzhou, Zhejiang 310003, China

Haihong Zhu ( $\nabla$ zhuhh72@zju.edu.cn )

Zhejiang University https://orcid.org/0000-0002-5387-8832

\section{Primary research}

Keywords: HCC, DNMT1, m5C modification patterns, TME, prognosis

Posted Date: June 8th, 2021

DOl: https://doi.org/10.21203/rs.3.rs-580707/v1

License: (a) (1) This work is licensed under a Creative Commons Attribution 4.0 International License. Read Full License 


\section{Abstract}

Background: 5-Methylcytosine $\left(\mathrm{m}^{5} \mathrm{C}\right)$ plays essential roles in hepatocellular carcinoma (HCC), but the association between $\mathrm{m}^{5} \mathrm{C}$ regulation and immune cell infiltration in $\mathrm{HCC}$ has not yet been clarified.

Methods: In this study, we analysed 371 patients with HCC from The Cancer Genome Atlas (TCGA) database, and the expression of $13 \mathrm{~m}^{5} \mathrm{C}$ regulators was investigated. Additionally, gene set variation analysis (GSVA), unsupervised clustering analysis, single-sample gene set enrichment analysis (ssGSEA), correlation analysis, and immunohistochemical $(\mathrm{IHC})$ staining were performed.

Results: Among the 371 patients, 41 had mutations in $\mathrm{m} 5 \mathrm{C}$ regulators, the frequency of which was $11.26 \%$. Then, we identified three $\mathrm{m} 5 \mathrm{C}$ modification patterns that had obvious tumour microenvironment (TME) cell infiltration characteristics. Cluster-1 had an immune rejection phenotype; Cluster-2 had an immunoinflammatory phenotype; and Cluster-3 had an immune desert phenotype. In addition, we found that DNMT1 was highly expressed in tumour tissues compared with normal tissues in a tissue microarray (TMA) and that it was positively correlated with many TME-infiltrating immune cells. High expression of the $\mathrm{m} 5 \mathrm{C}$ regulator DNMT1 was related to a poor prognosis in patients with HCC. Furthermore, we developed three Immu-clusters that were consistent with the immune characteristics of the $\mathrm{m} 5 \mathrm{C}$ methylation modification patterns. We also discovered differences in the levels of immune cells and expression of chemokines and cytokines among the three Immu-clusters.

Conclusions: Our work revealed the association between $\mathrm{m} 5 \mathrm{C}$ modification and immune regulators in the TME. These findings also suggest that DNMT1 has great potential as a prognostic biomarker and therapeutic target for HCC.

\section{Background}

Hepatocellular carcinoma (HCC) is the sixth most common cancer and the fourth leading cause of cancer-related death worldwide [1]. Risk factors for HCC include hepatitis B virus (HBV), hepatitis C virus $(\mathrm{HCV})$, non-alcoholic fatty liver disease, obesity with diabetes, etc. Patients who are infected with HCV can be treated with antiviral therapies, while patients who are infected with HBV remain infected throughout life [2]. The survival of patients is driven by tumour stage, with a 5-year survival rate exceeding $70 \%$ for those with early-stage HCC compared to a median survival time of 1-1.5 years for those with advancedstage HCC [3]. Most HCC patients are diagnosed at advanced stages, and limited effective therapeutic strategies are available [4].

Tumour cells are the driving cause of tumour development and progression. However, without the tumour microenvironment (TME), tumour cells cannot act alone in the progression of cancer. The TME includes the surrounding blood vessels, fibroblasts, immune cells, extracellular matrix and signalling molecules. These elements contribute to the processes of carcinogenesis and progression, while it is still a major challenge to fully evaluate the complex TME [5]. 
More than 150 RNA modifications have been found, such as 5-methylcytosine $\left(m^{5} \mathrm{C}\right)$, N1methyladenosine $\left(\mathrm{m}^{1} \mathrm{~A}\right)$, 5-hydroxymethylcytosine (hm5C), pseudouridine $(\Psi)$, N6-methyladenosine $\left(\mathrm{m}^{6} \mathrm{~A}\right)$ and other types of RNA modifications [6, 7]. Previous studies have mainly focused on m6A modification in regulating coding and non-coding RNA processing and function [8]. Emerging evidence has revealed the important role of RNA m5C in posttranscriptional regulation [9]. In addition, $\mathrm{m}^{5} \mathrm{C}$ modification was found to be abundant in mammalian cells, characterized by the addition of a methyl group at the carbon5 position of the cytosine base [10]. $\mathrm{m}^{5} \mathrm{C}$ is mainly distributed in GC-rich areas. Over 10000 potential sites of $\mathrm{m}^{5} \mathrm{C}$ modification have been detected in the whole human transcriptome [11]. RNA $\mathrm{m}^{5} \mathrm{C}$ is a dynamic process controlled by three major regulators, termed "writers" (add a special modification), "readers" (identify and bind modified nucleotides), and "erasers" (remove a special modification) [12].

Recently, targeting the TME has been an encouraging method for cancer treatment [13]. Some studies showed a correlation between RNA modification and TME-infiltrating immune cells [14-17]. However, due to technological limitations, the research above was restricted to one or two RNA modification regulators or cell types, while anti-tumour effects involve multiple tumour suppressors interacting in a vitally cooperative way. Hence, a deep understanding of TME cell infiltration mediated by several regulators of RNA modifications will help to enhance the perception of TME immune regulation, especially $\mathrm{m}^{5} \mathrm{C}$ modification.

In this study, we analysed 371 patients with HCC from The Cancer Genome Atlas (TCGA) database, and the samples were integrated to evaluate $\mathrm{m}^{5} \mathrm{C}$ modification patterns. Correlation analysis was performed between the $\mathrm{m}^{5} \mathrm{C}$ modification pattern and TME cell infiltration characteristics. Three different $\mathrm{m}^{5} \mathrm{C}$ modification patterns were discovered. Surprisingly, a high consistency was detected in TME characteristics under these three patterns, namely, the immune desert phenotype, immune-inflamed phenotype and immune-excluded phenotype, indicating that $\mathrm{m}^{5} \mathrm{C}$ modification plays an essential role in forming an individual TME.

\section{Methods}

\section{HCC data source and preprocessing}

Gene expression and clinical annotation data were downloaded from the TCGA database. Patients without complete survival data were excluded. The TCGA-Liver Hepatocellular Carcinoma (TCGA-LIHC) dataset was used for further analysis. Finally, a total of 371 patient in the TCGA-LIHC cohort were selected for this study.

For the TCGA dataset, the R package TCGAbiolinks [18], which was developed to analyse Genomic Data Commons (GDC) data, was utilized to download the fragments per kilobase per million mapped reads (FPKM) values of gene expression from the GDC (https://portal.gdc.cancer.gov). FPKM values were further converted to transcripts per kilobase million (TPM) values. Batch effects generated by factors 
unrelated to any biological variations were corrected for using the parametric and non-parametric empirical Bayes framework algorithm from the sva package. Data related to somatic mutations were downloaded from the TCGA database. R (3.6.1) together with Bioconductor packages were employed in the study.

\section{Unsupervised clustering analysis of $\mathrm{m}^{5} \mathrm{C}$ regulators}

A total of $13 \mathrm{~m}^{5} \mathrm{C}$ regulators were extracted from 371 patients in the TCGA-LIHC cohort: 11 writers (NOP2, NSUN2, NSUN3, NSUN4, NSUN5, NSUN6, NSUN7, DNMT1, TRDMT1, DNMT3A, DNMT3B), 1 eraser (TET2) and 1 reader (ALYREF). Unsupervised clustering analysis was employed to distinguish different $\mathrm{m}^{5} \mathrm{C}$ modifications, after which the classification of patients was conducted for subsequent analysis.

A consensus clustering algorithm [19] was employed to assure the number of clusters and their stability. The ConsensusClusterPlus package was applied to execute the workflow mentioned above, and the stability of the classification was accomplished by conducting 1000 repetitions [20].

\section{Gene set variation analysis (GSVA) and functional annotation}

To explore the disparity of biological processes in $\mathrm{m}^{5} \mathrm{C}$ modification patterns, the "GSVA" R package was used to perform GSVA. This package is based on a non-parametric and unsupervised algorithm and is widely used to estimate the variation in gene set enrichment in expression datasets [21]. GSVA was implemented with "c2.cp.kegg.v6.2.symbols" gene sets obtained from the Molecular Signatures Database (MSigDB). An adjusted $P$ value of less than 0.05 was regarded as statistically significant. We applied the "ClusterProfiler" $\mathrm{R}$ package to functionally annotate $\mathrm{m}^{5} \mathrm{C}$-related genes under the false discovery rate (FDR) threshold of $<0.05$.

\section{Single-sample gene set enrichment analysis (ssGSEA)}

The sSGSEA algorithm was used to determine the relative richness in cell infiltration in the TME. We obtained the gene set associated with each infiltrating immune cell type in the TME from Charoentong, who stores information on various human immune cells, including CD8 T cells, dendritic cells (DCs), natural killer (NK) T cells, macrophages, regulatory T cells, etc. [22, 23]. ssGSEA was employed to determine the enrichment scores and define the relative abundance of each TME-infiltrating cell type in the corresponding sample.

\section{Identification of differentially expressed genes (DEGs) among the $\mathrm{m} 5 \mathrm{C}$ phenotypes}

With the aim of distinguishing $\mathrm{m}^{5} \mathrm{C}$-related genes, all the patients were divided into three $\mathrm{m}^{5} \mathrm{C}$ modification patterns according to the expression of $\mathrm{m}^{5} \mathrm{C}$ regulators. The empirical Bayesian algorithm under the limma package in $\mathrm{R}$ was used to assure DEGs in heterogeneous modification patterns. 


\section{Correlation between the $\mathrm{m}^{5} \mathrm{C}$ gene signature and biological pathways}

A set of genes was constructed by Mariathasan et al. [24-26], in which genes associated with certain biological processes are stored. Correlation analysis was employed to explore the association between the gene signature of $\mathrm{m}^{5} \mathrm{C}$ and biological pathways.

\section{Immunohistochemical (IHC) staining}

Human HCC tissue arrays and normal tissues (catalogue number: HLivH180Su15) were purchased from Shanghai Outdo Biotech Co., Ltd. (Shanghai, P.R. China). The method of IHC staining has been reported previously. Briefly, antigen retrieval was performed by heating the tissue sections at $100^{\circ} \mathrm{C}$ for $30 \mathrm{~min}$ in target retrieval solution. Then, the tissue microarray (TMA) was incubated with a DNMT1 primary antibody ([EPR18453] (ab188453) Abcam, Cambridge, MA), followed by incubation with an anti-rabbit secondary antibody. Two independent pathologists blindly assessed the $\mathrm{IHC}$ results according to the staining area and intensity [27].

\section{Statistical analysis}

Spearman and distance correlation analyses were performed to obtain the correlation coefficients of the TME-infiltrating immune cells and the corresponding expression of $\mathrm{m}^{5} \mathrm{C}$ regulators. One-way analysis of variance (ANOVA) and Kruskal-Wallis tests were performed to compare differences between three or more groups [28]. The Kaplan-Meier method was utilized to generate survival curves for the prognostic analysis, and the log-rank test was applied to identify significant differences. Univariate Cox regression was adopted to determine the hazard ratios of $\mathrm{m}^{5} \mathrm{C}$ regulators and genes related to specific $\mathrm{m}^{5} \mathrm{C}$ phenotypes. Multivariable Cox regression was utilized to identify independent prognostic risk factors. Patients with complete relevant data were subjected to further analysis with a multivariate model. The multivariate results were visualized with the forestplot package in R. Copy number variations (CNVs) in $13 \mathrm{~m}^{5} \mathrm{C}$ regulators were plotted with the RCircos package [29]. All $P$ values were two-sided, with $p<0.05$ considered statistically significant. The analysis was accomplished in R 3.6.1 software.

\section{Results}

\section{Landscape of genetic variations in $\mathrm{m}^{5} \mathrm{C}$ regulators in $\mathrm{HCC}$}

Ultimately, 11 writers, 1 eraser and 1 reader were identified. First, the incidence of CNVs and somatic mutations in regulators in HCC were summarized. In 364 samples, 41 showed mutations in $\mathrm{m}^{5} \mathrm{C}$ regulators, the occurrence of which was $11.26 \%$. DNMT1 was found to be exposed to a higher frequency of mutations, followed by DNMT3A, while ALYREF, NSUN2, NSUN3, and NSUN5 were not (Fig. 1a). CNVs were also detected in 13 other regulators upon exploration of their modification frequencies. Most of the modifications involved a copy number expansion, but TET2, NOP2, and NSUN4 had a broad occurrence 
of deletions (Fig. 1b). The chromosome sites of the $\mathrm{m}^{5} \mathrm{C}$ regulators are shown in Fig. $1 \mathrm{c}$. Based on the expression of $13 \mathrm{~m}^{5} \mathrm{C}$ regulators in $\mathrm{HCC}$ patients, $\mathrm{HCC}$ samples could be thoroughly differentiated from normal samples (Fig. 1d). To determine whether the expression of $m^{5} \mathrm{C}$ regulators was influenced by the genetic mutations mentioned above, the mRNA expression of regulators was explored. We found that a change in $\mathrm{m}^{5} \mathrm{C}$ was an important factor leading to perturbations in the expression of $\mathrm{m}^{5} \mathrm{C}$ regulators. Compared with normal hepatic tissues, the expression of $\mathrm{m} 5 \mathrm{C}$ regulators with a CNV expansion was significantly higher than that in HCC tissues (e.g., ALYREF and NSUN2) (Fig. 1b and e). The analyses above showed that the genetic and expression alteration landscape of $\mathrm{m} 5 \mathrm{C}$ regulators in normal tissues and $\mathrm{HCC}$ tissues is highly heterogeneous, suggesting that the expression imbalance of $\mathrm{m}^{5} \mathrm{C}$ regulators plays an important role in $\mathrm{HCC}$ occurrence and progression.

\section{$\mathrm{m}^{5} \mathrm{C}$ methylation alteration patterns mediated by 13 regulators}

Univariate Cox regression analysis showed that $13 \mathrm{~m} 5 \mathrm{C}$ modulators have prognostic significance in HCC patients (Fig. 2a). The m5C regulator network revealed m5C modulator interactions, modulator connections and their prognostic significance for patients (Fig. 2b). The R package

ConsensusClusterPlus was applied to classify patients with qualitatively different $\mathrm{m}^{5} \mathrm{C}$ alteration patterns according to the expression of $13 \mathrm{~m}^{5} \mathrm{C}$ regulators, and unsupervised clustering analysis was performed to identify a total of 3 different modification patterns (120 cases in modification pattern 1, 178 cases in modification pattern 2, and 73 cases in modification pattern 3; referred to as m5C Clusters 1-3, respectively) (Fig. 2c and Table S1). The prognostic analysis of the three major m5C modification subtypes showed that Cluster-2 had a clear survival advantage over the others (Fig. 2d). The above results indicate that the crosstalk between erasers, readers and writers may play an important role in $\mathrm{m}^{5} \mathrm{C}$ alteration patterns and TME cell infiltration characteristics between individual tumours.

\section{TME cell infiltration characteristics in different $\mathrm{m}^{5} \mathrm{C}$ modification patterns}

To investigate the biological actions associated with $\mathrm{m}^{5} \mathrm{C}$ modification patterns, GSVA was conducted. As shown in Fig. 2e and Table S2, $\mathrm{m}^{5} \mathrm{C}$ Cluster-2 was remarkably enriched in the activation of stroma and carcinogenesis pathways, such as the ERBB signalling pathway, cell cycle signalling pathway, and cell adhesion pathway. Cluster-1 was associated with enrichment pathways (Fig. 2e). Cluster-3 was highly associated with carcinogenic activation (Fig. 2f). Unexpectedly, further analysis of TME cell infiltration showed that Cluster-1 was significantly enriched in the infiltration of innate immune cells, including eosinophils, NK cells, macrophages, CD8 T cells, mast cells, and active B cells (Fig. 3a). Compared with patients in Cluster-3, patients in Cluster-2 showed a corresponding survival advantage (Fig. 2d). Prior research has shown that tumours with an immune rejection phenotype exhibit large amounts of immune cells, and these immune cells are in the matrix around the tumour cell nest instead of inside the tissue 
[30]. GSVA showed that the modification of Cluster-1 was significantly related to matrix activation. Therefore, it was speculated that the Cluster- 1 matrix serves as an activation inhibitor of the anti-tumour effect of immune cells. Further analysis showed that matrix activity was greatly upgraded, activating the angiogenesis pathway. These results supported our hypothesis (Fig. 3b). Based on the above analysis, we found that the $3 \mathrm{~m}^{5} \mathrm{C}$ modification patterns had obvious TME cell infiltration characteristics. Cluster- 1 had an immune rejection phenotype, featuring natural infiltrating immune cells and an activated matrix; Cluster-2 had an immunoinflammatory phenotype, featuring adaptively infiltrated immune cells and immune activation; and Cluster-3 had an immune desert phenotype, featuring immune suppression.

\section{The $\mathrm{m} 5 \mathrm{C}$ regulator DNMT1 has a strong relationship with infiltrating immune cells}

To further explore the role of each $\mathrm{m} 5 \mathrm{C}$ regulator in the TME, Spearman correlation analysis was applied to examine the correlation between each TME-infiltrating cell type and $\mathrm{m}^{5} \mathrm{C}$ regulators (Fig. $4 \mathrm{a}$ ). An emphasis was placed on the regulator DNMT1, an m5C methyltransferase, and we revealed its positive relationship with the infiltration of many TME immune cells. An estimation method was applied to determine the expression of DNMT1 and the infiltration of immune cells. The results showed that higher DNMT1 expression was related to a higher immune score, which means that a TME with high DNMT1 expression has significantly high immune cell infiltration (Fig. 4b). Based on these results, the specific differences in 23 TME-infiltrating immune cells were explored between patients with high and low DNMT1 expression. We found that tumours exhibiting high DNMT1 expression had markedly more infiltration of 13 TME immune cells than those exhibiting low expression (Fig. 4c). Recently, attention was drawn to the regulatory mechanisms of $\mathrm{m}^{5} \mathrm{C}$ modification on the activation of DCs, which are the bridge connecting innate immunity with adaptive immunity, the activation of which depends on upregulating the expression of MHC molecules, adhesion molecules and costimulatory molecules (Fig. 4d). As expected, subsequent enrichment analysis showed that tumours with high DNMT1 expression showed remarkable enrichment in immune activation pathways (Fig. 4e). Therefore, it was speculated that $\mathrm{m}^{5} \mathrm{C}$ methylation modification mediated by DNMT1 may contribute to activated DCs in the TME, thus promoting the anti-tumour immune response in $\mathrm{HCC}$.

High expression of the $\mathrm{m} 5 \mathrm{C}$ regulator DNMT1 in tumour tissues is related to a poor prognosis in patients with HCC

IHC staining was used to determine the expression pattern of DNMT1 on a TMA consisting of 90 pairs of HCC tissues and adjacent tissues. Representative micrographs illustrate the various degrees of DNMT1 expression (Fig. 5a and b). The expression of DNMT1 was higher in tumour tissues than in control tissues (Fig. 5c), which was consistent with the findings in the TCGA-LIHC cohort (Fig. 1e). The correlation of DNMT1 expression with the clinicopathological characteristics of patients with HCC is shown in Table S3. In addition, Kaplan-Meier curve analysis showed that patients with high DNMT1 expression had shorter overall survival (OS) than those with low DNMT1 expression (Fig. 5d). Univariable 
and multivariable Cox regression analyses were used to determine whether the expression of DNMT1 was an independent risk factor. The univariable analysis revealed that DNMT1 expression was associated with tumour size and TB, AFP, and PD-L1 levels ( $<<0.05$, Table S4). Further analysis demonstrated that DNMT1 might serve as a prognostic predictor for HCC.

\section{Generation of the $\mathrm{m}^{5} \mathrm{C}$ gene signature and functional annotation}

For subsequent exploration of the biological behaviour of each $\mathrm{m}^{5} \mathrm{C}$ modification pattern, we ascertained $307 \mathrm{~m}^{5} \mathrm{C}$ phenotype-related DEGs with the limma package (Fig. 6a). clusterProfiler was employed to implement enrichment analysis on the DEGs. Table S5 summarizes the significantly enriched pathways. As expected, we detected enrichment in biological processes that are notably related to $\mathrm{m} 5 \mathrm{C}$ modification and immunity, which verified the important role that $\mathrm{m} 5 \mathrm{C}$ modification plays in immune regulation in the TME (Fig. 6b).

To further explain the association, we performed unsupervised clustering analysis to classify $307 \mathrm{~m}^{5} \mathrm{C}$ phenotype-related genes and extracting 27 immune-related genes: VIPR2, CCL7, RBP2, SLC10A2, FGF5, DEFA5, HTR3A, TRH, LCN15, AMBN, ADIPOQ, FGF3, CCK, NTF4, NDP, FGF9, PF4, CMA1, SFTPA2, CGB8, DEFA6, PF4V1, IL25, GH2, FGF8, SST and IAPP. Furthermore, we performed unsupervised clustering analysis based on these genes to categorize patients into different subtypes (Figure S1a-d). In line with the clustering analysis of $\mathrm{m}^{5} \mathrm{C}$ modification patterns, unsupervised clustering analysis revealed three different $\mathrm{m}^{5} \mathrm{C}$-modified phenotypes termed Immu-clusters $1-3$, respectively. Thus, there are 3 different distinct immune-related $\mathrm{m}^{5} \mathrm{C}$ methylation patterns. We observed that tumours in Immu-clusters 2 and 3 were associated with poor differentiation and enriched in diffuse histological subtypes. The opposite pattern was observed in Immu-Cluster 1. Patients whose survival status was known were mainly concentrated in Immu-cluster 1, while patients in clinical stage IV or with a high TNM grade were mainly concentrated in Immu-cluster 2 (Fig. 6c). The analysis also showed that three different gene clusters had different feature genes (Fig. 6c). In total, 114 of the 317 HCC patients clustered in Immu-cluster 1, which was associated with a better prognosis. The prognosis of patients in Immu-cluster 1 (110 patients) and immu-cluster 3 (93 patients) was poor (Fig. 6d). In the three immune clusters, a significant distinction in the expression of $\mathrm{m} 5 \mathrm{C}$ regulatory factors emerged. This result was consistent with the $\mathrm{m} 5 \mathrm{C}$ methylation modification patterns (Fig. 6e).

\section{Clinical and transcriptional features of the $\mathrm{m}^{5} \mathrm{C}$-related phenotypes}

To further explain the role that m5C-related phenotypes play in TME immune regulation, the levels of immune cells and expression of chemokines and cytokines in the three Immu-clusters were examined. The chosen cytokines and chemokines were taken from previously existing studies. Our analysis showed that activated CT4 T cells, immature B cells, regulatory T cells, NK cells, macrophages, mast cells, 
myeloid-derived suppressor cells (MDSCs), monocytes, $n$ neutrophils and plasmacytoid DCs were significantly different among the Immu-clusters (Fig. 7a). Tumour necrosis factor, interferon, CD8A, CXCL9, CXCL10, GZMA, GZMB, PRF1 and TBX2 were associated with immune activation transcription (Fig. 7b) [23, 31]. PD-L1, CD80, CD86, CTLA-4, HAVCR2, etc., were thought to be related to the transcription of immune checkpoints. We compared the transcription of these immune checkpoint genes in the three Immu-clusters (Fig. 7c). ACTA2, CLDN3, VIM, COL4A1, SMAD9, TWIST1, TGFBR2, TGRB1 and ZEB1 are related to the transcription of growth factor $\beta$ /EMT pathway transformation and exhibited significant differences between the three Immu-clusters (Fig. 7d). We found that mRNAs related to the TGF- $\beta / E M T$ pathway were significantly upregulated in Immu-cluster 2 and Immu-cluster 3 , indicating that this cluster is the matrix-activated group. Immu-cluster 1 showed elevated expression of mRNAs related to activated immune transcription. The above results indicated that Immu-cluster 1 could be categorized into an immune-activated group. The above results also showed that $\mathrm{m} 5 \mathrm{C}$ modification has a non-negligible regulatory effect on the formation of different TMEs.

\section{Discussion}

According to previous reports, tumours, including $\mathrm{HCC}$, are mainly driven by genetic mutations. In recent years, epigenetic modifications have been found to play a critical role in the carcinogenesis and molecular pathogenesis of $\mathrm{HCC}[8,9] . \mathrm{m} 5 \mathrm{C}$ is the most preventative and best understood DNA modification in eukaryotes [32]. In recent years, emerging evidence has revealed the important role of RNA $\mathrm{m} 5 \mathrm{C}$ in posttranscriptional regulation. Several studies have revealed that $\mathrm{m} 5 \mathrm{C}$ regulators and $\mathrm{m} 5 \mathrm{C}$ methylation play essential roles in different cancer types, including HCC. He et al. [33] found that ALYREF and NSUN4 could be promising targets for HCC therapies. In addition, studies showed the map of m5C methylation based on HCC tissues and paired non-tumour tissues at the mRNA, IncRNA, and circRNA levels [34-36]. Recent studies showed that NSUN2 could promote tumour progression in HCC [37] and gastric cancer [38]. Similar to our findings, Xue et al. [39, 40] found that DNMT1 played important roles in head and neck squamous cell carcinoma.

Recently, increasing evidence has shown interactions between the tumour immune -microenvironment (TIME) and RNA modifications. Yi et al. [17] reported that copy number alterations in m6A methylation regulators affected immune cell infiltration in head and neck squamous cell carcinoma. Lin and colleagues also attempted to explore the relationship between $\mathrm{m} 6 \mathrm{~A}$ regulators and tumour-infiltrating immune cells by sSGSEA in glioma. Shen et al. [16] found that m6A modification patterns were correlated with immune regulation in $\mathrm{HCC}$ and might provide novel immune therapeutic targets. However, as an important posttranscriptional modification, the role of RNA m5C methylation in the immune regulation of $\mathrm{HCC}$ is still unclear. Here, we described the TME cell infiltration characteristics in different $\mathrm{m} 5 \mathrm{C}$ modification patterns. Furthermore, we identified 3 distinct immune-related m5C methylation subtypes and investigated the levels of immune cells and expression of chemokines and cytokines in the three Immu-clusters. All the results indicate that the generation of immune-related $\mathrm{m} 5 \mathrm{C}$ methylation subtypes contribute to understanding the molecular mechanisms of $\mathrm{HCC}$ and provide novel clues for predicting the prognosis of patients with HCC. 
It has been demonstrated that DNMT1 is an essential methyltransferase for the maintenance of DNA methylation. Previous evidence has shown that DNMT1 is overexpressed in breast cancer [41], thyroid cancer cells [42], and pancreatic cancer [43]. Furthermore, high DNMT1 expression is significantly associated with a poor prognosis $[44,45]$. Consistent with our results, we found that DNMT1 expression was increased in tumour tissues compared with normal tissues in the TMA and TCGA cohort. In our study, Kaplan-Meier curve analysis and univariable and multivariable Cox regression analysis further demonstrated that the expression of DNMT1 is an independent risk factor for HCC. Therefore, DNMT1 might serve as a promising prognostic predictor and therapeutic target for HCC.

\section{Conclusions}

Taken together, our results showed the association between $\mathrm{m} 5 \mathrm{C}$ modification and distinct immune phenotypes. Moreover, we found a key m5C modification regulator, DNMT1, which has great potential as a prognostic biomarker and therapeutic target for HCC.

\section{Abbreviations}

m5C, 5-Methylcytosine; HCC, hepatocellular carcinoma; TCGA, The Cancer Genome Atlas ; GSVA, gene set variation analysis; SSGSEA, single-sample gene set enrichment analysis; IHC, immunohistochemical; TME, tumour microenvironment; TMA,tissue microarray; HBV, hepatitis B virus; $\mathrm{HCV}$, hepatitis $\mathrm{C}$ virus; m1A, N1methyladenosine; hm5C, 5-hydroxymethylcytosine; $\Psi$, pseudouridine; m6A, N6-methyladenosine; GDC, Genomic Data Commons; FPKM, fragments per kilobase per million mapped reads; TPM, transcripts per kilobase million; GSVA, Gene set variation analysis; MSigDB, Molecular Signatures Database; FDR, false discovery rate; DCs, dendritic cells; NK, natural killer; DEGs, differentially expressed genes; ANOVA, oneway analysis of variance; CNVs, copy number variations; OS, overall survival; MDSCs, myeloid-derived suppressor cells; TIME, tumour immune-microenvironment.

\section{Declarations}

\section{Ethical approval and consent to participate}

The study has been approved by the institutional ethical committee and all patients have signed the informed consent.

\section{Consent for publication}

Consent to publish was obtained from all authors.

\section{Availability of data and materials}

All data in our study are available upon request. 
The authors declare no competing interests.

\section{Funding}

This study was supported by grants awarded by the National Science and Technology Major Project of China (NO 2018ZX10302206), Science and Technology Major Projects of Zhejiang Province (NO 2018C04016), and the Science and Technology Major Projects of Ningbo (NO 2016C51008).

\section{Authors' Contributions}

XG, HZ and QC have equal contributions to this study. XGand HZ designed the whole study. QZ and JW conducted the statistical analysis. XG and QC draft the manuscript. QC made the relevant edits to the manuscript. XG and $\mathrm{HZ}$ revised the manuscript. All authors read and approved the final manuscript.

\section{Acknowledgements}

Not applicable.

\section{References}

1. Llovet JM, Kelley RK, Villanueva A, Singal AG, Pikarsky E, Roayaie S, et al. Hepatocellular carcinoma. Nat Rev Dis Primers. 2021;7:6.

2. European Association for the Study of the Liver. EASL 2017 clinical practice guidelines on the management of hepatitis B virus infection. J Hepatol. 2017;67:370-98.

3. Llovet JM, Zucman-Rossi J, Pikarsky E, Sangro B, Schwartz M, Sherman M, et al. Hepatocellular carcinoma. Nat Rev Dis Primers. 2016;2:16018.

4. Hernandez-Gea V, Toffanin S, Friedman SL, Llovet JM. Role of the microenvironment in the pathogenesis and treatment of hepatocellular carcinoma. Gastroenterology. 2013;144:512-27.

5. Hanahan D, Coussens LM. Accessories to the crime: functions of cells recruited to the tumor microenvironment. Cancer Cell. 2012;21:309-22.

6. Davalos V, Blanco S, Esteller M. SnapShot: messenger RNA modifications. Cell. 2018;174:498-e1.

7. García-Vílchez R, Sevilla A, Blanco S. Post-transcriptional regulation by cytosine-5 methylation of RNA. Biochim Biophys Acta Gene Regul Mech. 2019;1862:240-52.

8. Nombela P, Miguel-López B, Blanco $\mathrm{S}$. The role of $\mathrm{m}^{6} \mathrm{~A}, \mathrm{~m}^{5} \mathrm{C}$ and $\Psi$ RNA modifications in cancer: novel therapeutic opportunities. Mol Cancer. 2021;20:18.

9. Xue C, Zhao Y, Li L. Advances in RNA cytosine-5 methylation: detection, regulatory mechanisms, biological functions and links to cancer. Biomark Res. 2020;8:43.

10. Bestor TH. Cloning of a mammalian DNA methyltransferase. Gene. 1988;74:9-12.

11. Bourgeois G, Ney M, Gaspar I, Aigueperse C, Schaefer M, Kellner S, et al. Eukaryotic rRNA modification by yeast 5-methylcytosine-methyltransferases and human proliferation-associated 
antigen p120. PLoS One. 2015;10:e0133321.

12. Yang Y, Hsu PJ, Chen YS, Yang YG. Dynamic transcriptomic m(6)A decoration: writers, erasers, readers and functions in RNA metabolism. Cell Res. 2018;28:616-24.

13. Bejarano L, Jordāo MJC, Joyce JA. Therapeutic targeting of the tumor microenvironment. Cancer Discov. 2021;11:933-59.

14. Zhang B, Wu Q, Li B, Wang D, Wang L, Zhou YL. m(6)A regulator-mediated methylation modification patterns and tumor microenvironment infiltration characterization in gastric cancer. Mol Cancer. 2020;19:53.

15. Chong W, Shang L, Liu J, Fang Z, Du F, Wu H, et al. m(6)A regulator-based methylation modification patterns characterized by distinct tumor microenvironment immune profiles in colon cancer. Theranostics. 2021;11:2201-17.

16. Shen S, Yan J, Zhang Y, Dong Z, Xing J, He Y. N6-methyladenosine (m6A)-mediated messenger RNA signatures and the tumor immune microenvironment can predict the prognosis of hepatocellular carcinoma. Ann Transl Med. 2021;9:59.

17. Yi L, Wu G, Guo L, Zou X, Huang P. Comprehensive analysis of the PD-L1 and immune infiltrates of m(6)A RNA methylation regulators in head and neck squamous cell carcinoma. Mol Ther Nucleic Acids. 2020;21:299-314.

18. Colaprico A, Silva TC, Olsen C, Garofano L, Cava C, Garolini D, et al. TCGAbiolinks: an R/Bioconductor package for integrative analysis of TCGA data. Nucleic Acids Res. 2016;44:e71.

19. Hartigan JA, Wong MA. Algorithm AS 136: A K-means clustering algorithm. J R Stat Soc C (Appl Stat). 1979;28:100-8.

20. Wilkerson MD, Hayes DN. Consensusclusterplus: a class discovery tool with confidence assessments and item tracking. Bioinformatics. 2010;26:1572-3.

21. Hänzelmann S, Castelo R, Guinney J. GSVA: gene set variation analysis for microarray and RNA-seq data. BMC Bioinformatics. 2013;14:7.

22. Charoentong P, Finotello F, Angelova M, Mayer C, Efremova M, Rieder D, et al. Pan-cancer immunogenomic analyses reveal genotype-immunophenotype relationships and predictors of response to checkpoint blockade. Cell Rep. 2017;18:248-62.

23. Barbie DA, Tamayo P, Boehm JS, Kim SY, Moody SE, Dunn IF, et al. Systematic RNA interference reveals that oncogenic KRAS-driven cancers require TBK1. Nature. 2009;462:108-12.

24. Mariathasan S, Turley SJ, Nickles D, Castiglioni A, Yuen K, Wang Y, et al. TGF $\beta$ attenuates tumour response to PD-L1 blockade by contributing to exclusion of T cells. Nature. 2018;554:544-8.

25. Rosenberg JE, Hoffman-Censits J, Powles T, van der Heijden MS, Balar AV, Necchi A, et al. Atezolizumab in patients with locally advanced and metastatic urothelial carcinoma who have progressed following treatment with platinum-based chemotherapy: a single-arm, multicentre, phase 2 trial. Lancet. 2016;387:1909-20. 
26. Şenbabaoğlu Y, Gejman RS, Winer AG, Liu M, Van Allen EM, de Velasco G, et al. Tumor immune microenvironment characterization in clear cell renal cell carcinoma identifies prognostic and immunotherapeutically relevant messenger RNA signatures. Genome Biol. 2016;17:231.

27. Zhang JG, Zhou HM, Zhang X, Mu W, Hu JN, Liu GL, et al. Hypoxic induction of vasculogenic mimicry in hepatocellular carcinoma: role of HIF-1 a, RhoA/ROCK and Rac1/PAK signaling. BMC Cancer. 2020;20:32.

28. Hazra A, Gogtay N. Biostatistics series module 3: comparing groups: numerical variables. Indian J Dermatol. 2016;61:251-60.

29. Mayakonda A, Lin DC, Assenov Y, Plass C, Koeffler HP. Maftools: efficient and comprehensive analysis of somatic variants in cancer. Genome Res. 2018;28:1747-56.

30. Chen DS, Mellman I. Elements of cancer immunity and the cancer-immune set point. Nature. 2017;541:321-30.

31. Zeng D, Li M, Zhou R, Zhang J, Sun H, Shi M, et al. Tumor microenvironment characterization in gastric cancer identifies prognostic and immunotherapeutically relevant gene signatures. Cancer Immunol Res. 2019;7:737-50.

32. Piperi C, Papavassiliou AG. Strate-gies for DNA methylation analysis in developmental studies. Dev Growth Differ. 2011;53:287-99.

33. He Y, Yu X, Li J, Zhang Q, Zheng Q, Guo W. Role of m(5)C-related regulatory genes in the diagnosis and prognosis of hepatocellular carcinoma. Am J Transl Res. 2020;12:912-22.

34. Zhang Q, Zheng Q, Yu X, He Y, Guo W. Overview of distinct 5-methylcytosine profiles of messenger RNA in human hepatocellular carcinoma and paired adjacent non-tumor tissues. J Transl Med. 2020;18:245.

35. He Y, Zhang Q, Zheng Q, Yu X, Guo W. Distinct 5-methylcytosine profiles of circular RNA in human hepatocellular carcinoma. Am J Transl Res. 2020;12:5719-29.

36. He Y, Shi Q, Zhang Y, Yuan X, Yu Z. Transcriptome-wide 5-methylcytosine functional profiling of long non-coding RNA in hepatocellular carcinoma. Cancer Manag Res. 2020;12:6877-85.

37. Sun Z, Xue S, Zhang M, Xu H, Hu X, Chen S, et al. Aberrant NSUN2-mediated m(5)C modification of $\mathrm{H} 19$ IncRNA is associated with poor differentiation of hepatocellular carcinoma. Oncogene. 2020;39:6906-19.

38. Mei L, Shen C, Miao R, Wang JZ, Cao MD, Zhang YS, et al. RNA methyltransferase NSUN2 promotes gastric cancer cell proliferation by repressing p57(Kip2) by an m(5)C-dependent manner. Cell Death Dis. 2020;11:270.

39. Xue M, Shi Q, Zheng L, Li Q, Yang L, Zhang Y. Gene signatures of m5C regulators may predict prognoses of patients with head and neck squamous cell carcinoma. Am J Transl Res. 2020;12:6841-52.

40. Cui J, Zheng L, Zhang Y, Xue M. Bioinformatics analysis of DNMT1 expression and its role in head and neck squamous cell carcinoma prognosis. Sci Rep. 2021;11:2267. 
41. Wang P, Chu W, Zhang X, Li B, Wu J, Qi L, et al. Kindlin-2 interacts with and stabilizes DNMT1 to promote breast cancer development. Int J Biochem Cell Biol. 2018;105:41-51.

42. Zhang Y, Sun B, Huang Z, Zhao DW, Zeng Q. Shikonin Inhibites migration and invasion of thyroid cancer cells by downregulating DNMT1. Med Sci Monit. 2018;24:661-70.

43. Peng DF, Kanai Y, Sawada M, Ushijima S, Hiraoka N, Kosuge T, et al. Increased DNA methyltransferase 1 (DNMT1) protein expression in precancerous conditions and ductal carcinomas of the pancreas. Cancer Sci. 2005;96:403-8.

44. Li A, Omura N, Hong SM, Goggins M. Pancreatic cancer DNMT1 expression and sensitivity to DNMT1 inhibitors. Cancer Biol Ther. 2010;9:321-9.

45. Hong L, Sun G, Peng L, Tu Y, Wan Z, Xiong H, et al. The interaction between miR-148a and DNMT1 suppresses cell migration and invasion by reactivating tumor suppressor genes in pancreatic cancer. Oncol Rep. 2018;40:2916-25.

\section{Figures}


A

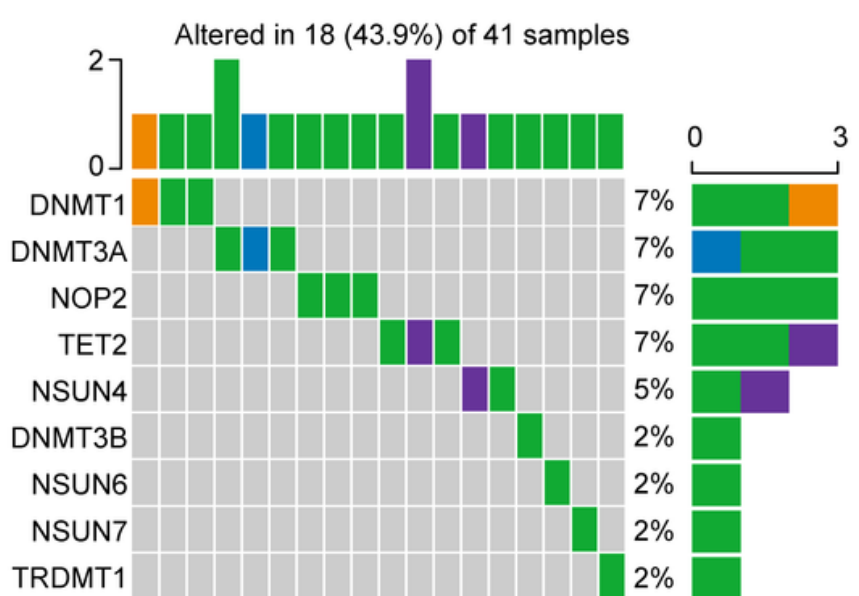

B

- Frame Shift Del - Frame Shift Ins

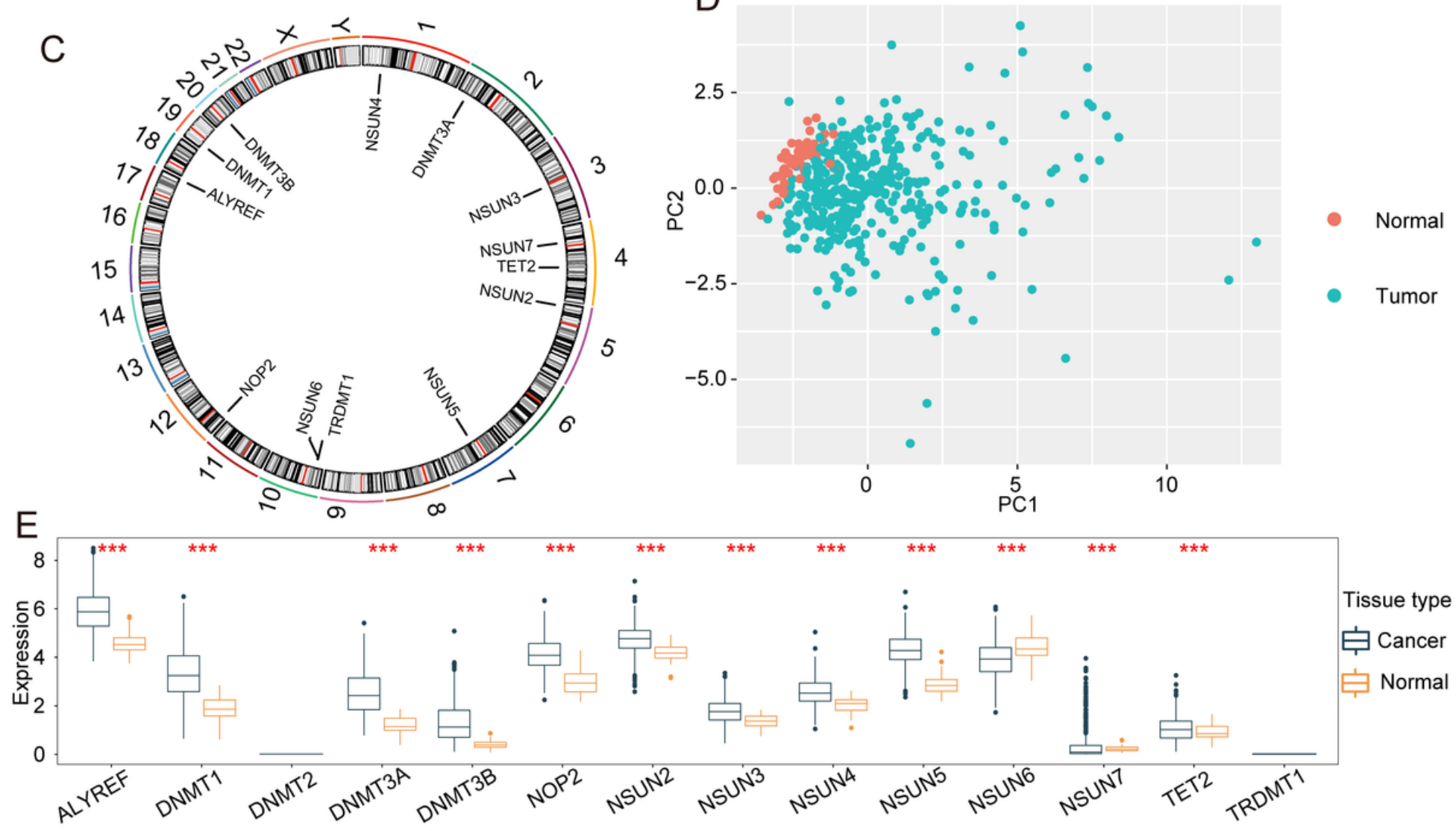

Figure 1

$\mathrm{CNV}$ s and somatic mutations in $13 \mathrm{~m} 5 \mathrm{C}$ regulators in HCC. a. Mutation frequencies of the top $9 \mathrm{~m} 5 \mathrm{C}$ regulators.b. CNV alterations among the 13 regulators. c. Locations of mutations in the $\mathrm{m} 5 \mathrm{C}$ regulators at the chromosome level. d. Principal component analysis was used to distinguish tumour tissues and normal tissues based on the expression of $\mathrm{m} 5 \mathrm{C}$ regulators.e. The expression profiles of $\mathrm{m} 5 \mathrm{C}$ regulator genes in tumour tissues and adjacent normal tissues. 
A

\begin{tabular}{|c|c|c|c|}
\hline & p-value & $\operatorname{HR}(95 \% \mathrm{Cl})$ & \\
\hline NSUN4 & $8.30 E-06$ & $1.5(1.3-1.9)$ & $\bullet-1$ \\
\hline DNMT3A & $3.30 \mathrm{E}-05$ & $1.2(1.1-1.4)$ & $\bullet-1$ \\
\hline DNMT1 & 4.10E-05 & 1.1(1.1-1.2) & $\bullet$ \\
\hline ALYREF & $4.50 E-05$ & $1(1-1)$ & • \\
\hline NOP2 & $2.00 E-04$ & $1.1(1-1.2)$ & $\bullet$ \\
\hline DNMT3B & $7.00 E-04$ & $1.3(1.1-1.4)$ & $\bullet$ \\
\hline NSUN2 & 0.014 & 1.1(1-1.1) & $\bullet$ \\
\hline NSUN5 & 0.015 & $1(1-1.1)$ & - \\
\hline TRDMT1 & 0.017 & $3.6(1.3-11)$ & $\bullet$ \\
\hline NSUN3 & 0.03 & $1.6(1-2.5)$ & $\bullet$ \\
\hline NSUN7 & 0.27 & $1.2(0.87-1.7)$ & $\bullet$ \\
\hline TET2 & 0.56 & $1.2(0.67-2.1)$ & $\bullet$ \\
\hline \multirow[t]{2}{*}{ NSUN6 } & 0.7 & $0.99(0.93-1.1)$ & - \\
\hline & & & 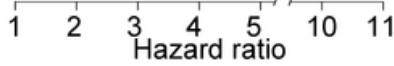 \\
\hline
\end{tabular}

\section{$\begin{array}{llll}\text { C } & \text { Cluster } 1 & \text { Cluster } 2 & \text { Cluster } 3\end{array}$}

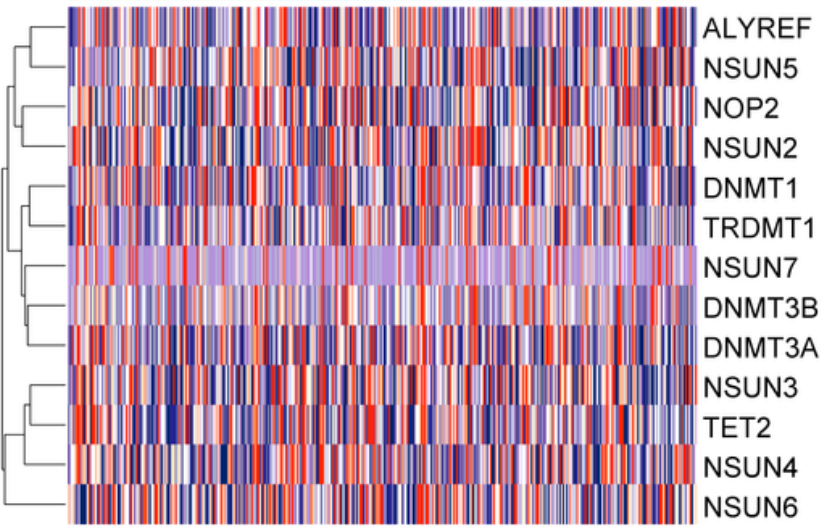

\section{E} Cluster $1 \quad$ Cluster 2

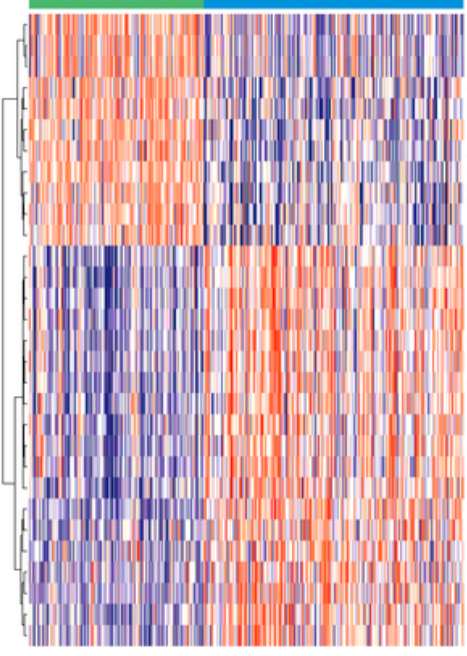

Cardiac muscle contraction Disease Cxidative phosphorylation cascades euroactive ligang receptor interaction Arachidonic acid metism Olfactory transduction Arginine and proline metabolism Nitrogen metabolism Non-small cell lung cancer Endometrial cancer

Prostate cancer

Cheromic myeloid leukemia Panreatic cancer Colorectal cancer Adherens junction nositol phosphate metabolism Neurotrophin signaling pathway Renal cell carcinoma MTOR signaling pathway Ubiquitin mediated proteolysis Basal transcription actors Non-homologous end joining Mismatch repair

Progesterone mediated oocyte maturation Oocyte meiosis
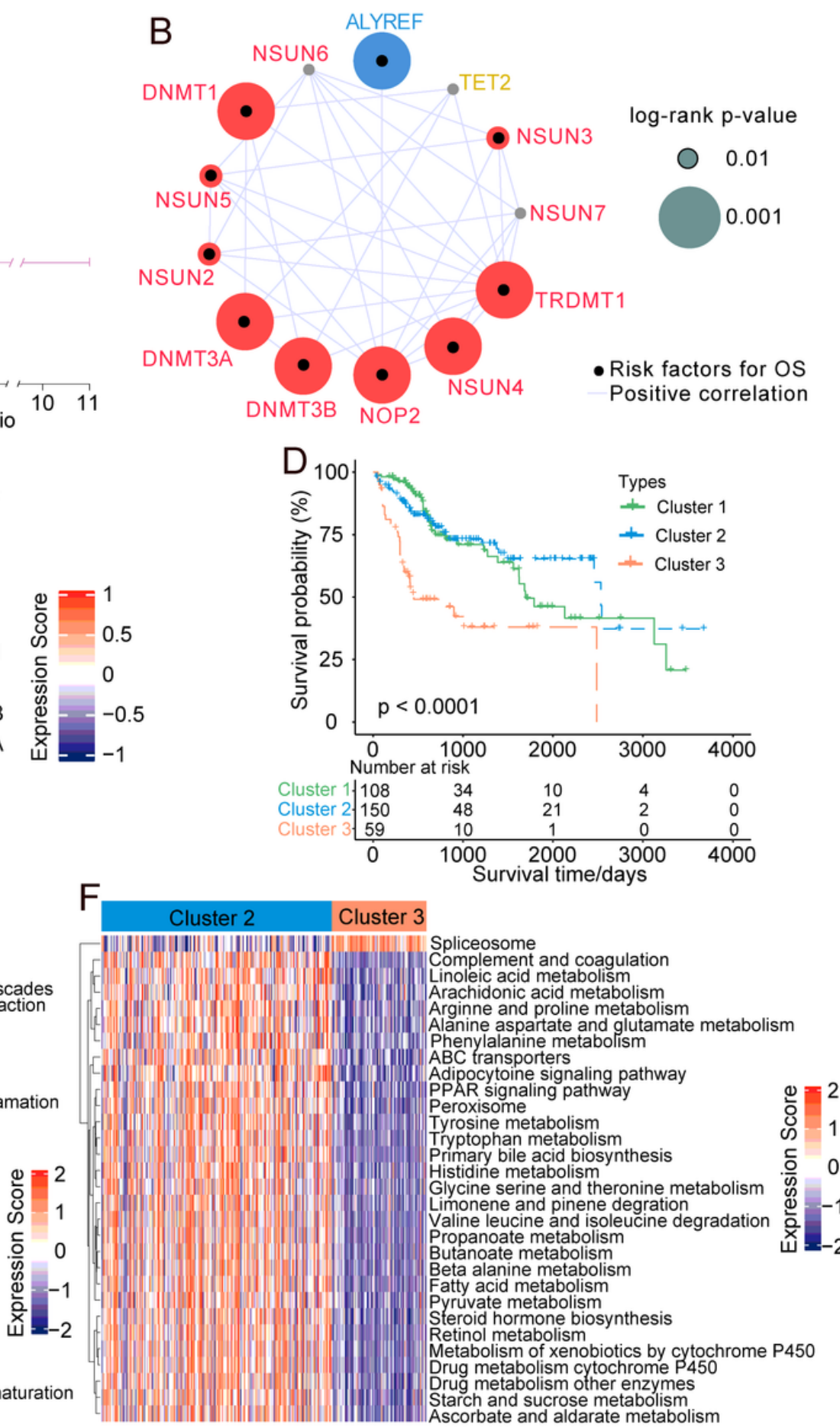

Figure 2

m5C methylation alteration patterns and related biological characteristics. a. Univariate Cox regression analysis of the $13 \mathrm{~m} 5 \mathrm{C}$ regulators in patients with HCC. b. The network of $\mathrm{m} 5 \mathrm{C}$ regulators and their prognostic significance for HCC patients. c. Unsupervised clustering analysis of $13 \mathrm{~m} 5 \mathrm{C}$ regulators in HCC. d. Survival analysis of HCC patients in the TCGA-LIHC cohort according to the three m5C clusters. ef.A heatmap of GSVA results shows the representative hallmark pathways associated with distinct m5C modification patterns. 


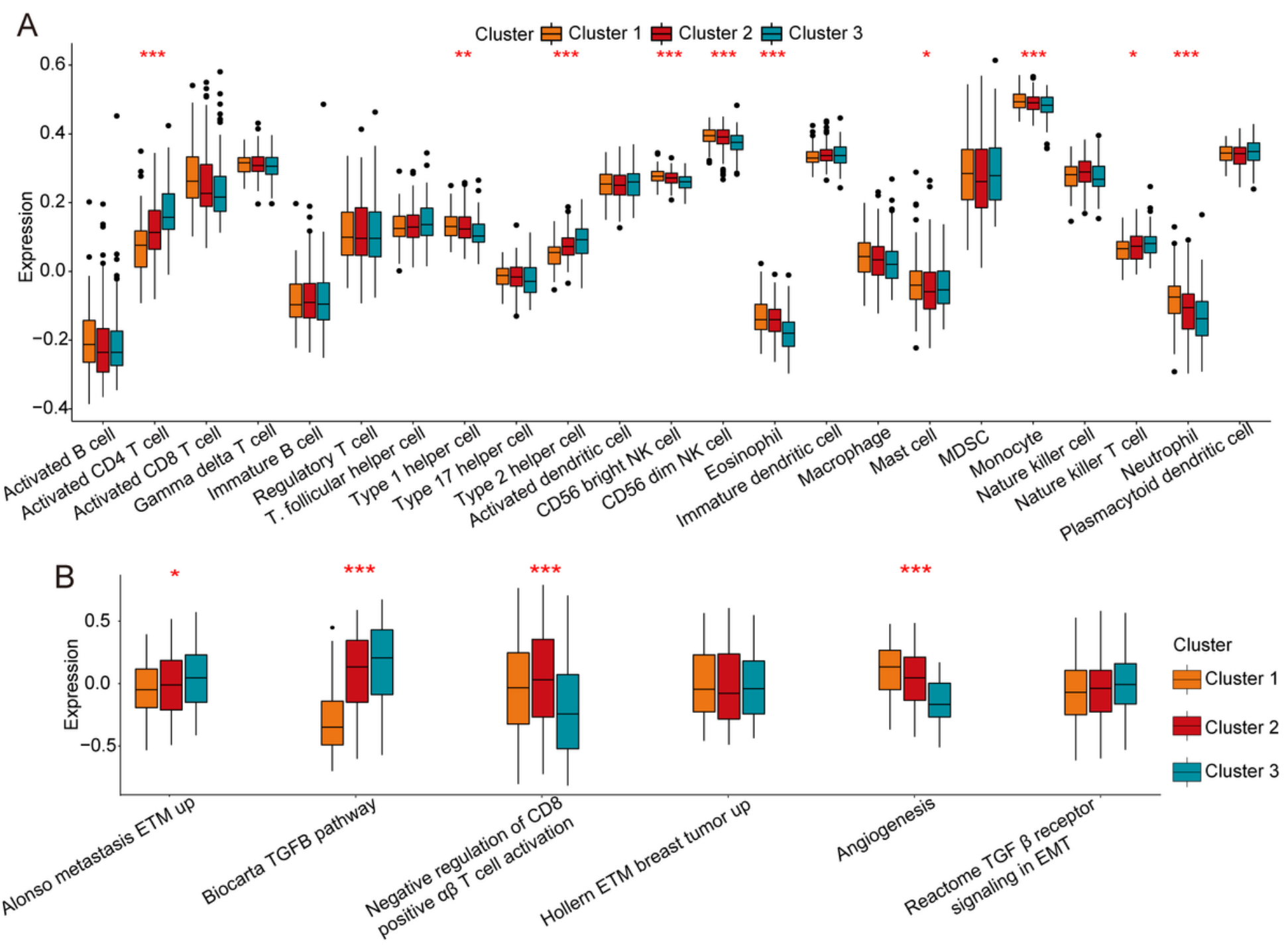

Figure 3

TME characteristics in different $\mathrm{m} 5 \mathrm{C}$ modification patterns. a Comparison of the abundance of immune infiltrating cells in three clusters. b. Differences in cellular biological pathways among the three clusters. 
A

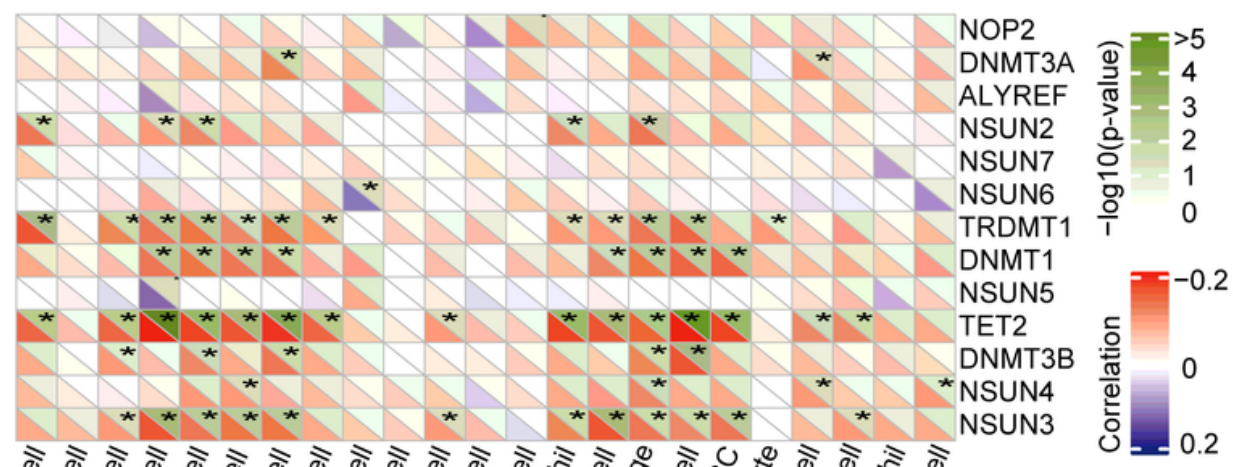

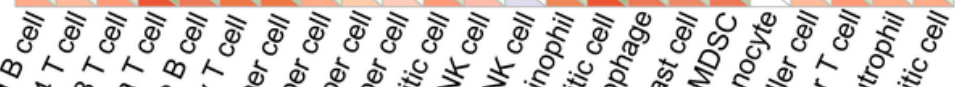

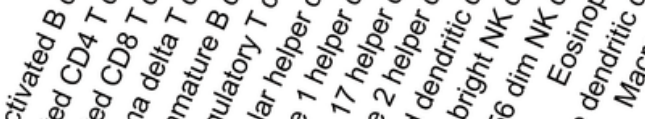

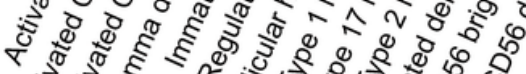

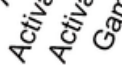
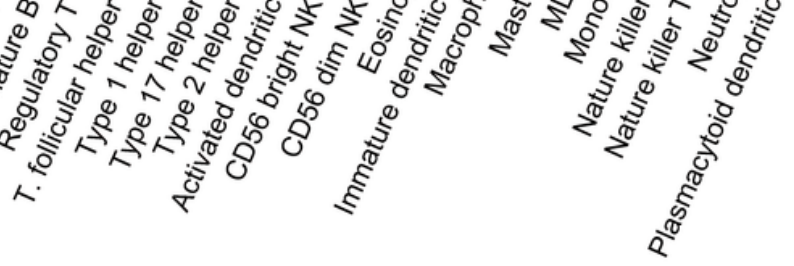

C
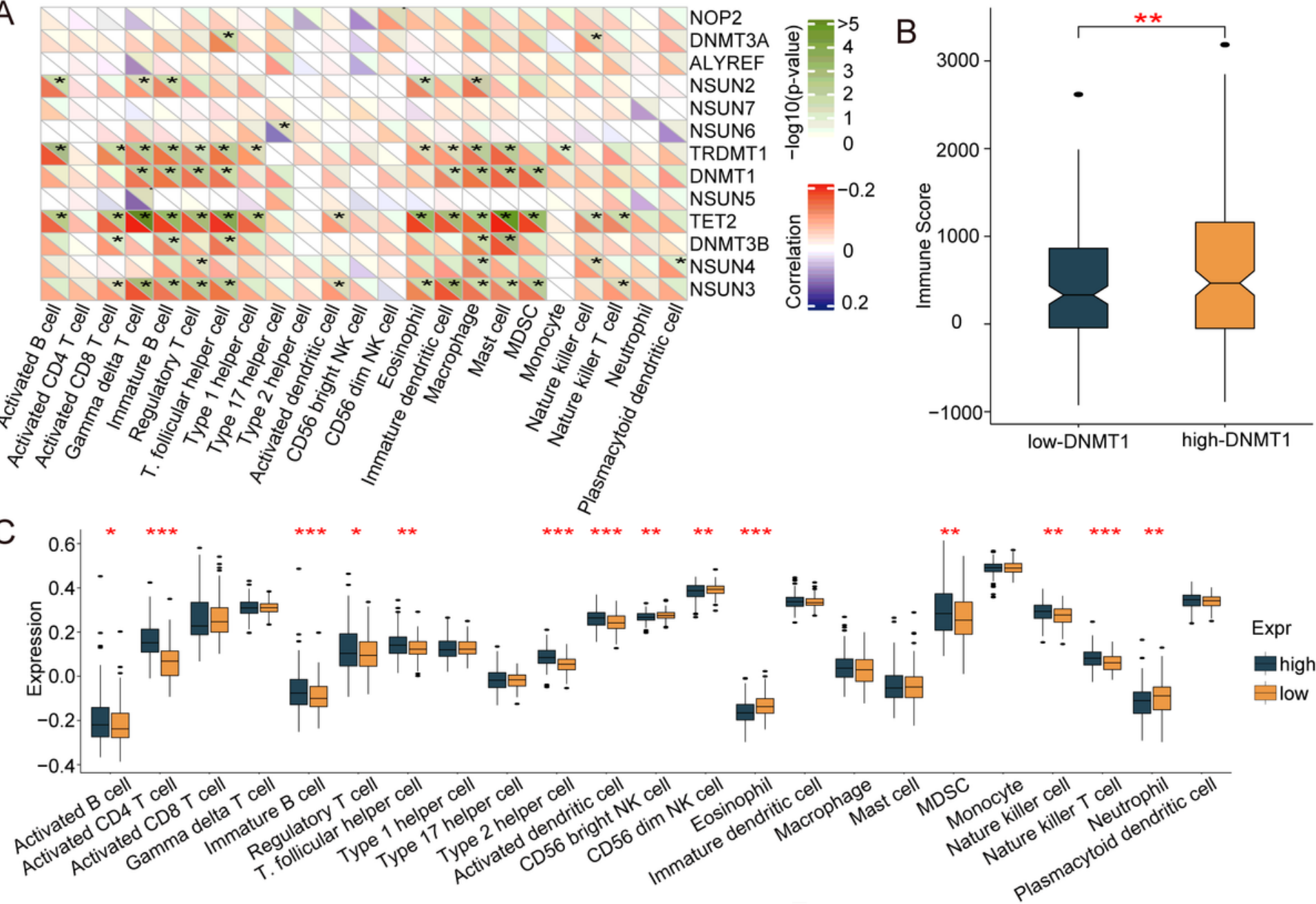

D Expr Ehigh
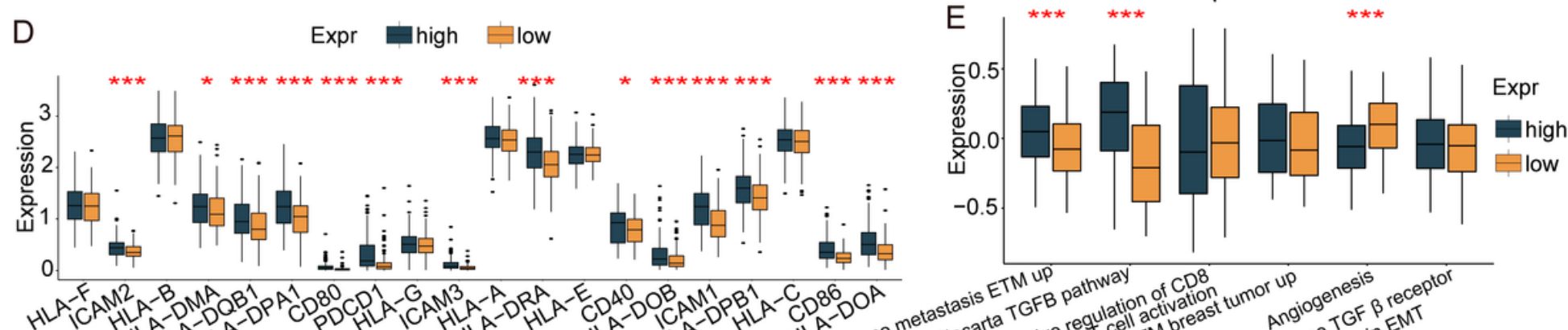

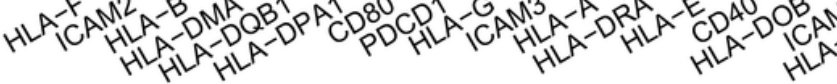

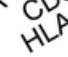

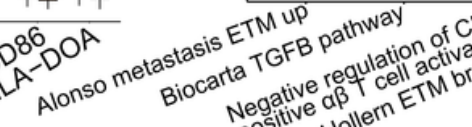

\section{Figure 4}

Association of TME-infiltrating cells with the $\mathrm{m} 5 \mathrm{C}$ regulator of DNMT1. a. Correlation between $\mathrm{m} 5 \mathrm{C}$ regulators and different immune cells using Spearman analysis. b. Immune scores of the low DNMT1 group and the high DNMT1 group. c. Comparison of the abundance of immune-infiltrating cells in the low DNMT1 group and high DNMT1 group. $d$. Correlation between $\mathrm{m} 5 \mathrm{C}$ regulators and the activation of dendritic cells. E. High DNMT1 expression shows significant enhancement of the immune-activated pathway. 
A

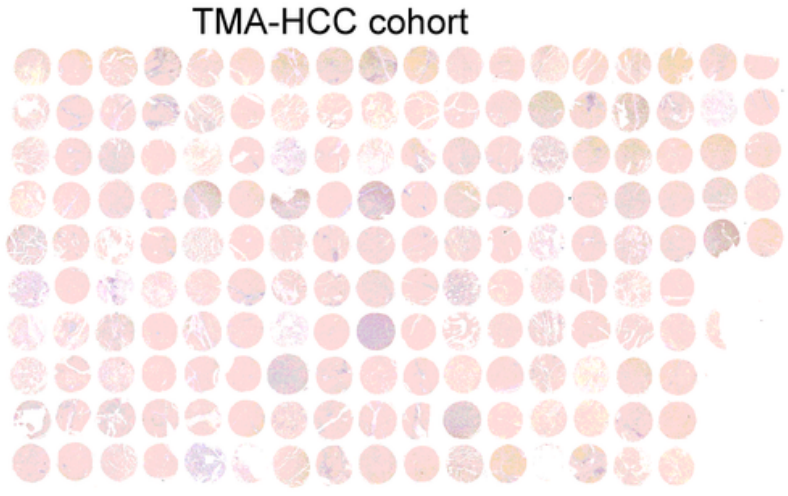

C

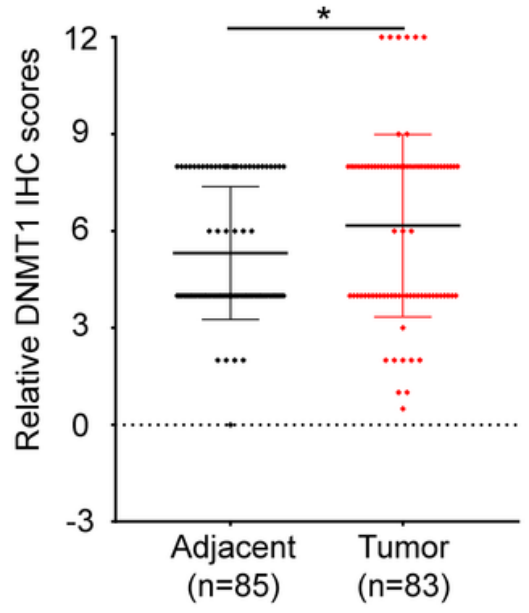

B
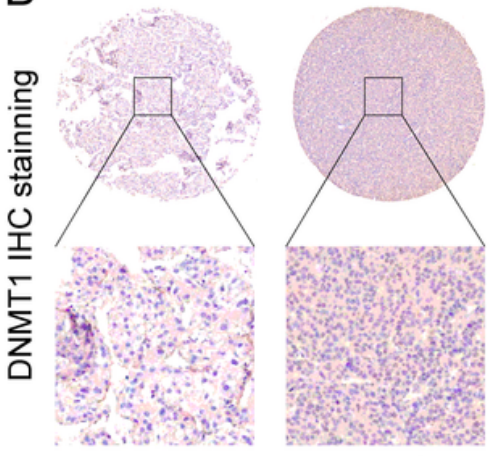

Level 1

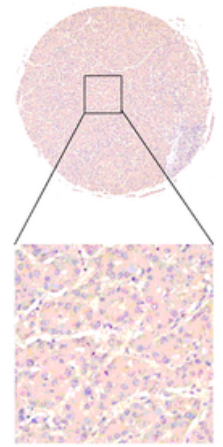

Level 2

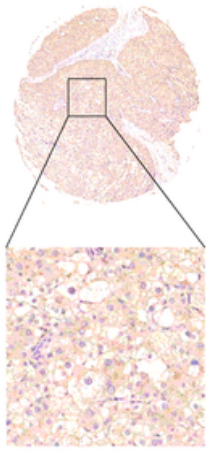

Level 3
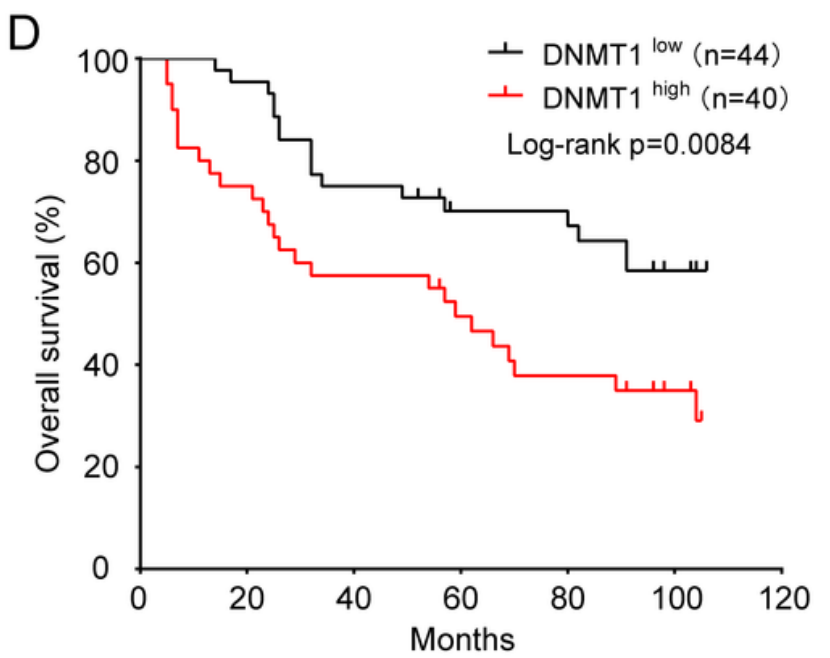

Figure 5

Expression of DNMT1 in human HCC tumour tissues and control tissues. a. Panoramic scanning of DNMT1 by IHC staining. b. Representative IHC staining of DNMT1 in samples. c. The expression of DNMT1 is higher in HCC tissues than in normal tissues. d. Kaplan-Meier analysis showed that patients with higher levels of DNMT1 had shorter OS times than those with low levels of DNMT1. 


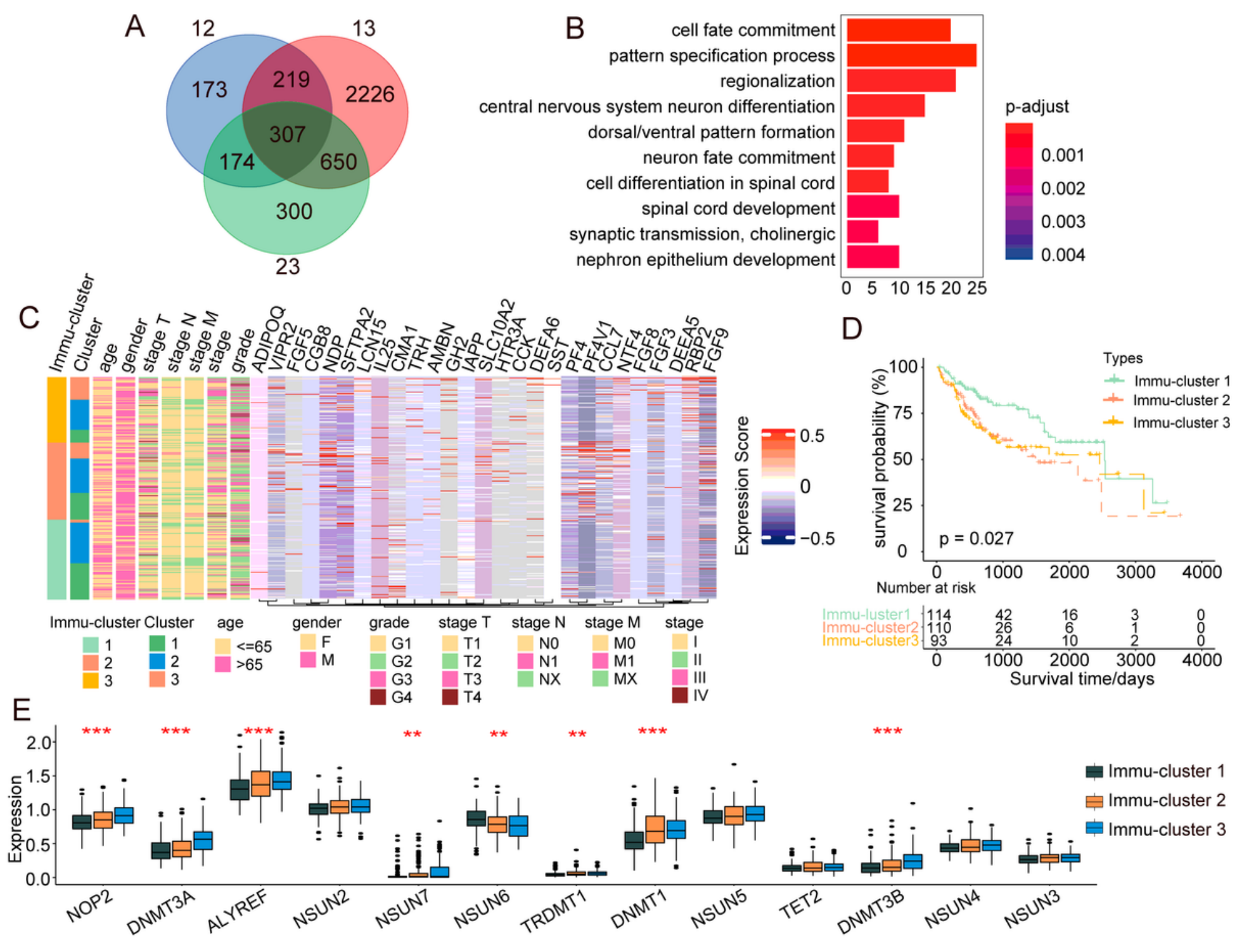

Figure 6

Identification of distinct Immu-clusters based on immune-related DEGs in m5C modification patterns.a. A total of 307 m5C-related DEGs between three m5Cclusters were identified, as shown in the Venn diagram. b. Enrichment of biological processes significantly related to DEGs. c. The selected genes were used to classify patients into different genomic subtypes by unsupervised clustering analysis. $d$. Kaplan-Meier curves indicated that the genomic subtypes were correlated with the prognosis of patients with HCC. e. Significant differences in the expression of $\mathrm{m} 5 \mathrm{C}$ regulators. 


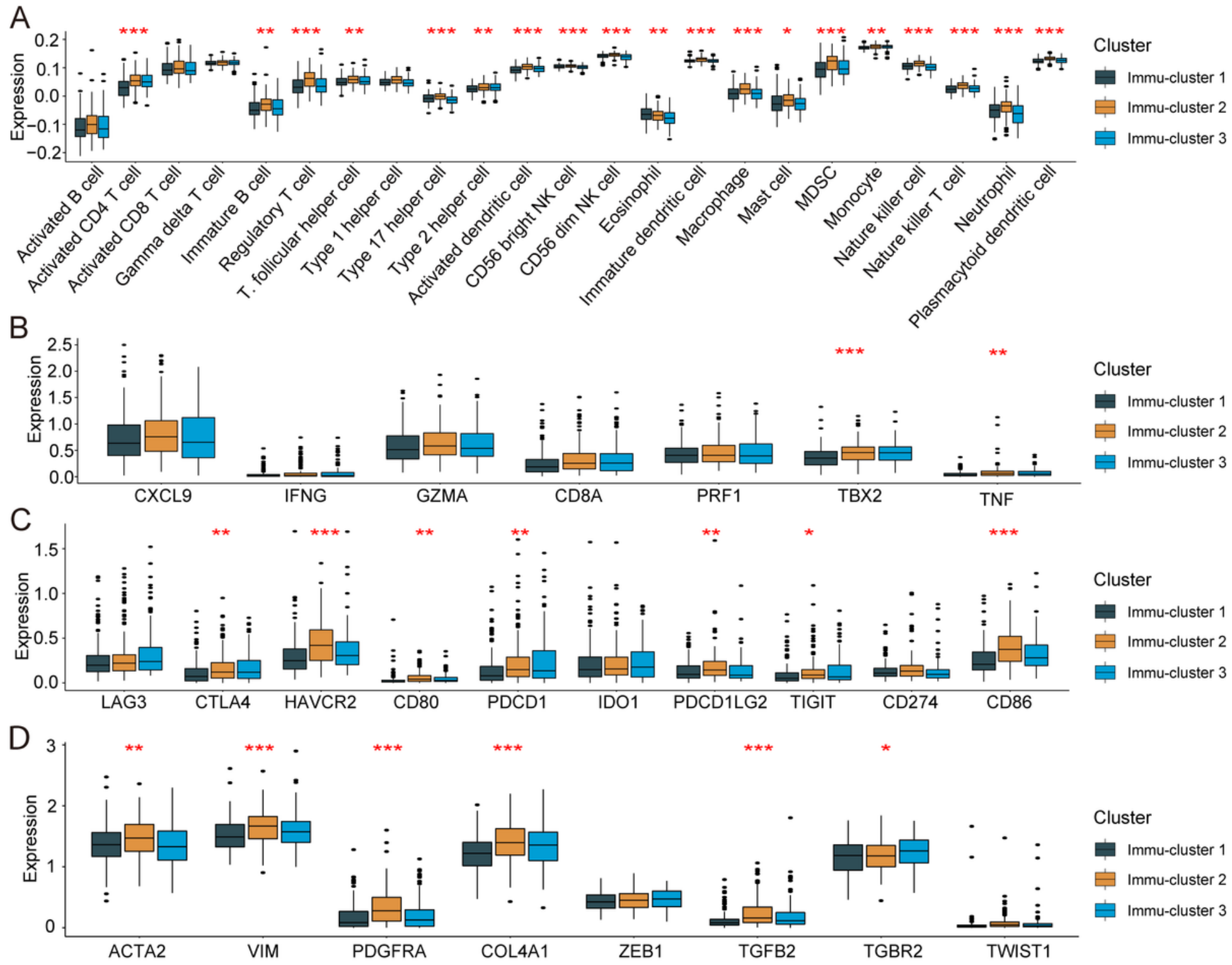

Figure 7

Association between the expression of $\mathrm{m} 5 \mathrm{C}$ regulators and immunoregulation in the TME. a. Differences in immune cell infiltration in the three Immu-clusters. b. Comparison of immune-related cytokine expression in the three Immu-clusters. c. Comparison of the transcription of immune checkpoint genes in the three Immu-clusters. d. Immu-clusters involved in the transcription of the TGF- $\beta$ /EMT pathway.

\section{Supplementary Files}

This is a list of supplementary files associated with this preprint. Click to download.

- FigureS1.tif

- TableS1.xlsx

- Tables2.xlsx 
- TableS3.docx

- Tables4.docx

- TableS5.xlsx 